\title{
$2-5-96$
}

SANDIA REPORT

SAND96-0022 • UC-706

Unlimited Release

Printed January 1996
Men

FEB 141996

OSTI

\section{Theoretical Description of Methodology in PHASER (Probabilistic Hybrid Analytical System Evaluation Routine)}

\section{J. Arlin Cooper}

\section{Prepared by}

Sandia National Laboratories

Albuquerque, New Mexico 87185 and Livermore, California 94550

for the United States Department of Energy

under Contract DE-AC04-94AL85000

Approved for public releasegtistribution is unlimited.
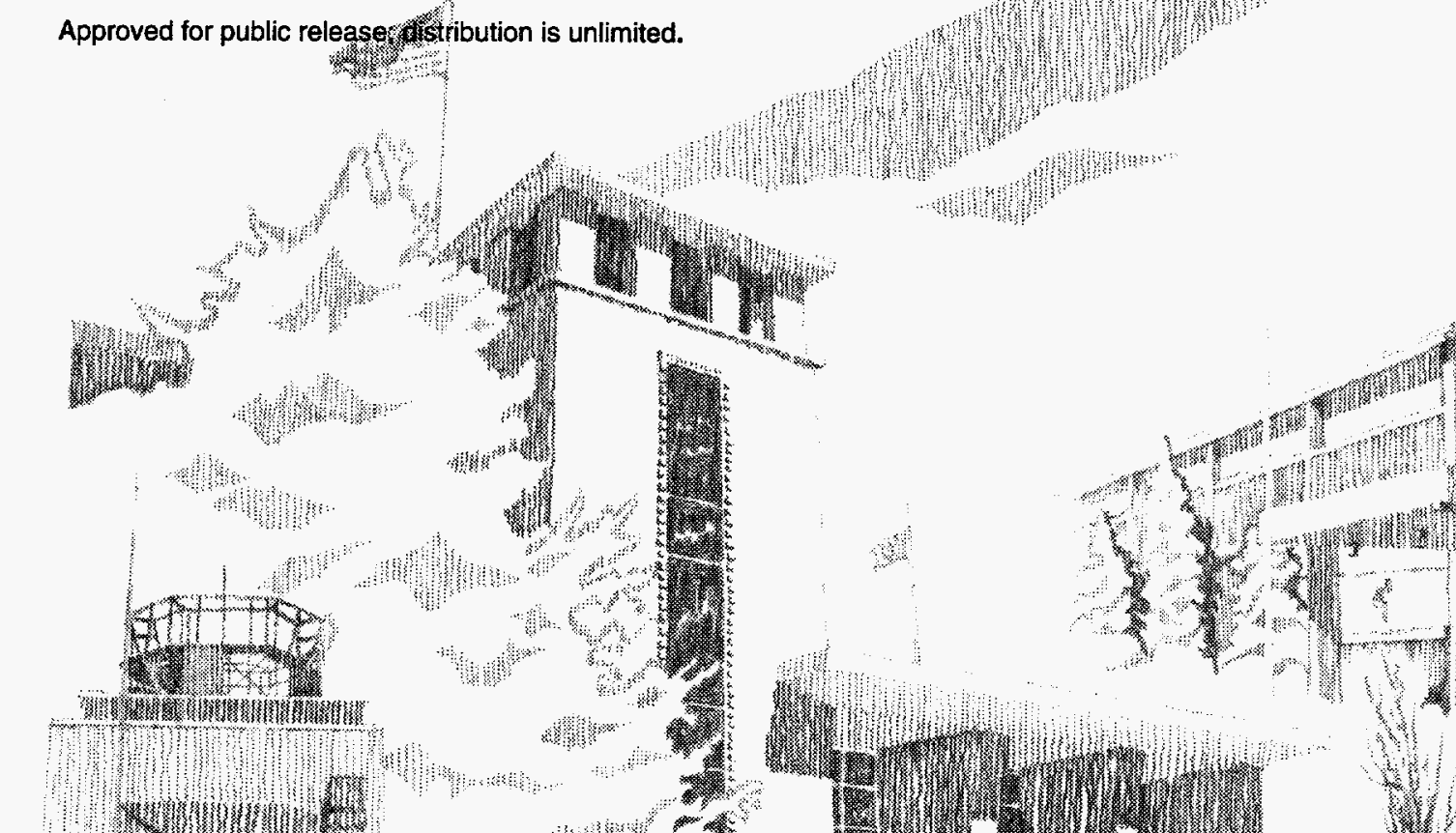

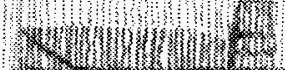


Issued by Sandia National Laboratories, operated for the United States Department of Energy by Sandia Corporation.

NOTICE: This report was prepared as an account of work sponsored by an agency of the United States Government. Neither the United States Government nor any agency thereof, nor any of their employees, nor any of their contractors, subcontractors, or their employees, makes any warranty, express or implied, or assumes any legal liability or responsibility for the accuracy, completeness, or usefulness of any information, apparatus, product, or process disclosed, or represents that its use would not infringe privately owned rights. Reference herein to any specific commercial product, process, or service by trade name, trademark, manufacturer, or otherwise, does not necessarily constitute or imply its endorsement, recommendation, or favoring by the United States Government, any agency thereof or any of their contractors or subcontractors. The views and opinions expressed herein do not necessarily state or reflect those of the United States Government, any agency thereof or any of their contractors.

Printed in the United States of America. This report has been reproduced directly from the best available copy.

Available to DOE and DOE contractors from Office of Scientific and Technical Information

PO Box 62

Oak Ridge, TN 37831

Prices available from (615) 576-8401, FTS 626-8401

Available to the public from

National Technical Information Service

US Department of Commerce

5285 Port Royal Rd

Springfield, VA 22161

NTIS price codes

Printed copy: A03

Microfiche copy: A01 


\section{DISCLAMMER}

Portions of this document may be illegible in electronic image products. Images are produced from the best available original document. 
SAND96-0022

Unlimited Release

Printed January 1996

Distribution

Category UC-706

\title{
Theoretical Description of Methodology in PHASER (Probabilistic Hybrid Analytical System Evaluation Routine)
}

\author{
J. Arlin Cooper \\ System Studies Department \\ Sandia National Laboratories \\ Albuquerque, NM 87185-0490
}

\begin{abstract}
$\underline{\text { Abstract }}$
Probabilistic safety analyses (PSAs) frequently depend on fault tree and event tree models, using probabilities of "events" for inputs. Uncertainty or variability is sometimes included by assuming that the input probabilities vary independently and according to an assumed stochastic probability distribution model. Evidence is accumulating that this methodology does not apply well to some situations, most significantly when the inputs contain a degree of subjectivity or are dependent.

This report documents the current status of an investigation into methods for effectively incorporating subjectivity and dependence in PSAs and into the possibility of incorporating inputs that are partly subjective and partly stochastic. One important byproduct of this investigation was a computer routine that combines conventional PSA techniques with newly developed subjective techniques in a "hybrid" (subjective and conventional PSA) program. This program (PHASER) and a user's manual are now available for beta use.
\end{abstract}




\section{Acknowledgments}

The PHASER code development was the responsibility of Bob Roginski, SNL Assessment Technology Department. In addition to accurately implementing the mathematical methodology, he meticulously incorporated numerous features for fast and efficient computation; and he produced a user-friendly product by taking great pains to incorporate flexibility for input options, simplicity of feedback from the program to the user, and diagnostic capabilities. Bob also collaborated in interactions about the theoretical methodology features described in this report.

Other collaborators on methodology included Dr. Scott Ferson, Applied Biomathematics, Prof. George Klir, State University of New York, Binghamton, Dr. Doug Cooper, Naval Research Laboratory, Prof. Tim Ross, University of New Mexico, and Prof. Lev

Ginzburg, State University of New York, Stony Brook. 


\section{TABLE OF CONTENTS}

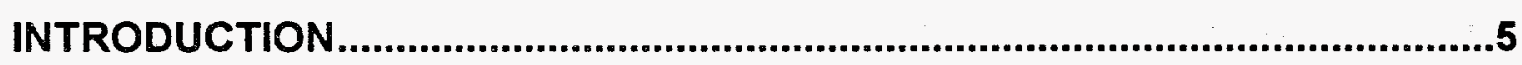

THE NEED FOR SUBJECTIVITY ABOUT INPUTS TO ANALYSES $\ldots . . . . . . . . . . . . . .5$

PROBABILISTIC CALCULUS VARIABILITY ANALYSES...............................6

FUZZY MATHEMATICS UNCERTAINTY ANALYSES.....................................

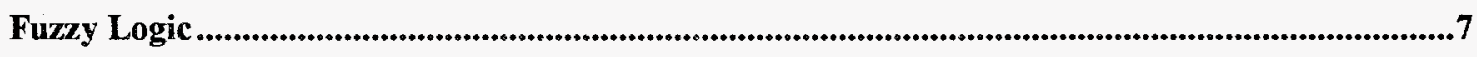

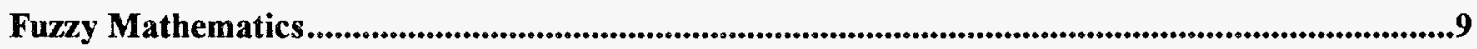

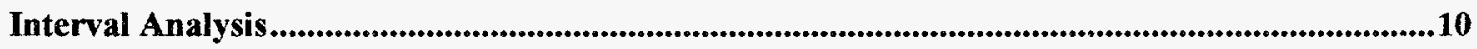

ANALYSIS OF EXTREMES...................................................................10

Conventional Analysis of Extremes................................................................................................11

Fuzzy Mathematics Analysis of Extremes...............................................................................11

HYBRID MATHEMATICS FOR VARIABILITY AND UNCERTAINTY ...............13

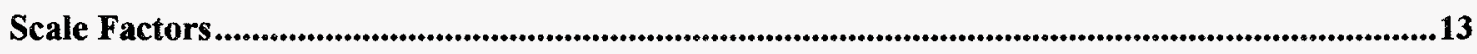

Confidence Factors ................................................................................................................................15

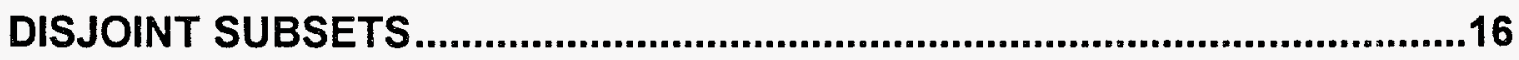

CONSTRAINED MATHEMATICS.................................................................19

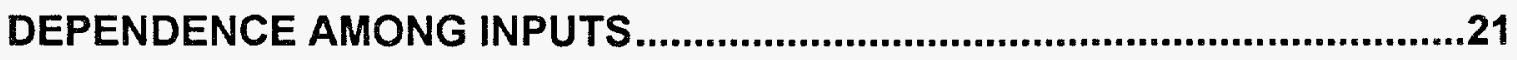

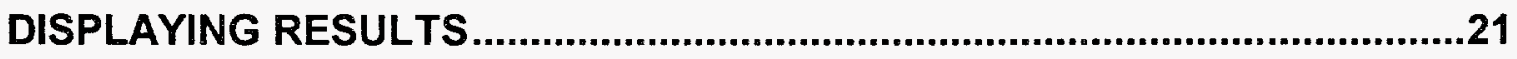

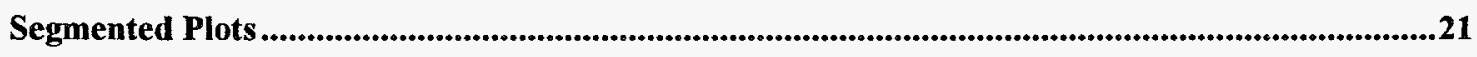

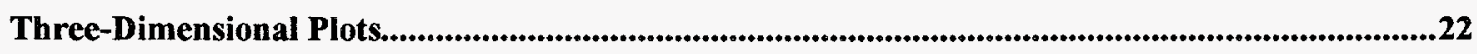

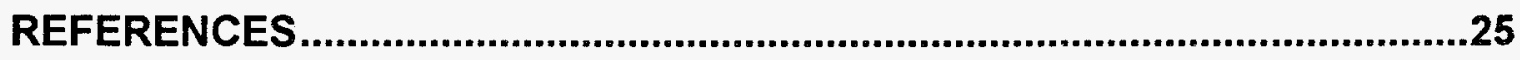




\section{List of Figures}

FIGURE 1. A SOLUTION FOR TENSILE FAILURE OF SERIES-PARALLEL CHAIN LINKS..............7

FIGURE 2. SUBJECTIVE FAIIURE CHARACTERISTICS OF INDIVIDUAL LINKS ..........................8

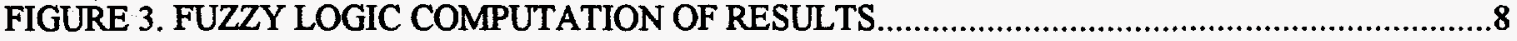

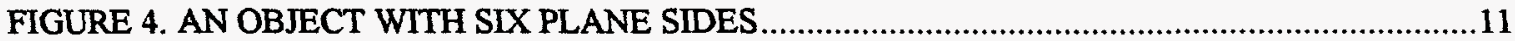

FIGURE 5. PDF FOR PROBABILITY OF THROWING FOUR ONES ...........................................12

FIGURE 6. SUBJECTIVE ESTIMATE OF PROBABILITY OF THROWING FOUR ONES .................12

FIGURE 7. A VISUAL DEPICTION OF A SCALED HYBRID NUMBER …......................................14

FIGURE 8. EXAMPLE OF CONSTRAINED MATHEMATICS RESULT FOR PROBABILITY OF

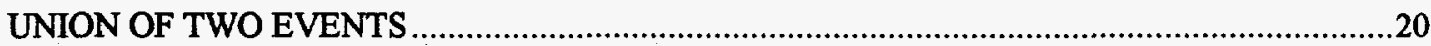

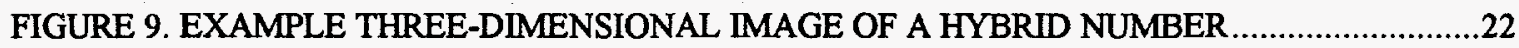

FIGURE 10. TWO-DIMENSIONAL REPRESENTATION OF FIG. 9 


\section{Introduction}

Probabilistic safety analyses (PSAs) are most frequently considered to be a quantitative determination of the probability of system risk (probability of safety failure), which inversely determines the level of system safety [Ref. 1]. These can depend on fault tree and/or event tree models, using probabilities for the tree inputs. Uncertainty or variability is sometimes included by assuming that the inputs vary independently according to an assumed (among dozens of possibilities) probability distribution model.

A somewhat focused view of safety analysis is that the important regime of interest in a spectrum of uncertainty is the extreme that indicates maximum potential loss of safety. The challenge is complicated by an accumulating collection of evidence that safety analyses, even those with a focus on extremes, do not reliably enough portray the chance of safety failure commensurate with the frequency of actual occurrences. There is considerable evidence [e.g., Refs. 2-6] that conventional methodology does not provide sufficient warnings in a variety of situations, most significantly when the inputs contain a degree of subjectivity or are dependent. One such area of interest (where it is crucial that no mistake be made) is abnormal-environment nuclear detonation weapon safety. This is the motivation that led to an investigation of methods for supplementing conventional PSA techniques with the aims of incorporating potential subjectivity and dependence in input information and of carefully studying the processes leading to extreme values in the resultant variations.

This report documents the current status of the effort. One important byproduct of the investigation is a computer routine that combines conventional PSA techniques with new subjective techniques in a "hybrid" (conventional PSA and subjective) program. This program (PHASER, or Probabilistic Hybrid Analytical Safety Evaluation Routine) is now available in Version 1.00 for beta users.

\section{The Need for Subjectivity about Inputs to Analyses}

When safety analysts are being trained in most university or short course programs, there is emphasis on prescribed processes (e.g., flipping coins, rolling dice, "normally" distributed inputs, etc.,) These techniques are firmly based on well understood theory and are accurate so long as the situation fits the model assumptions. Unfortunately, in analysis of real world systems safety, the situation does necessarily fit text-book models. Analogies are having bent coins (the bending characteristics only known qualitatively), nonrectilinear dice (face size and shape only known qualitatively), unknown probability distributions (statistical characteristics only known qualitatively), etc. Ideally, the characteristics of the bent coins, deformed dice, and process variability could be studied until there could be complete confidence in a model that fits the real situation. This is a good, but often impractical goal. 
There are many real-world examples of inputs to PSAs that are necessarily at least partly subjective. The concern is that modeling based on stochasticity may be merely an assertion, rather than a reflection of reality.

\section{Probabilistic Calculus Variability Analyses}

There are many potential sources of quantitative variation in analyses. One form is classical variability, which is applicable to prescribed problems such as those mentioned earlier involving dice and coins. Another form is subjective uncertainty, which means that available data are not definitive enough to prescribe the variations. Any mix of these two forms is possible. Since the terms variability and uncertainty, respectively, are now used in the literature extensively to describe these two restrictive sources of lack of definitive knowledge about any particular parameter [Ref. 7], these terms will be so used in this report. However, the goal is to accommodate any potential mix of variability and uncertainty, and this mix will also be termed "uncertainty."

In order to be general, variability will herein be considered stochastic, meaning that any sample can assume any value (statistically determined) over an interval, and that every sample is independent of every other sample ${ }^{1}$. The statistical determination can be described by, for example, a probability density function (pdf). The pdf can be one of the dozens of possible mathematically described pdfs or can be "customized" to the observed data.

Operations such as addition, subtraction, and multiplication can be performed on stochastic variables by applying probabilistic calculus [Ref. 8] to the corresponding pdfs. For example, the addition of two pdfs $f_{1}(x)$ and $f_{2}(x)$ can be described by the convolution integral:

$$
f(y)=\int_{-\infty}^{\infty} f_{1}(x) f_{2}(y-x) d x
$$

The product of two nonnegative pdfs, $f_{1}(x)$ and $f_{2}(x)$, is:

$$
f(y)=\int_{0}^{\infty} \frac{f_{1}(x) f_{2}(y / x) d x}{x} .
$$

In practice, the solution to these types of operations is usually accomplished through some form of Monte Carlo simulation. For example, a routine based on Latin Hypercube Sampling (LHS) is used at Sandia. LHS is more efficient than pure Monte Carlo analysis in determining an accurate statistical description of the result. PHASER uses LHS as an adjunct to solve the probabilistic calculus computations that are needed for the PHASER probabilistic results.

\footnotetext{
${ }^{1}$ Overcoming this restriction will be addressed in the section on input dependence.
} 


\section{Fuzzy Mathematics Uncertainty Analyses}

Pure subjective uncertainty necessarily is based on opinion, and since the results of any analysis are only as good as the analysis inputs, the best possible inputs come from estimators who have the most applicable expertise. There are many procedures in use for determining results based on subjective inputs, for example the Analytical Hierarchy Process (AHP) [Ref. 9]. Our investigation converged on fuzzy logic and fuzzy mathematics as having the most applicable input capabilities and mathematical processing bases.

\section{Fuzzy Logic}

The difference between fuzzy logic and fuzzy mathematics is roughly analogous to the difference between Boolean algebra and the algebra of real numbers. As an example, the solution for the tensile failure of two parallel chains, each having two links for which the individual link tensile strength is known subjectively, can be solved by fuzzy logic as follows:
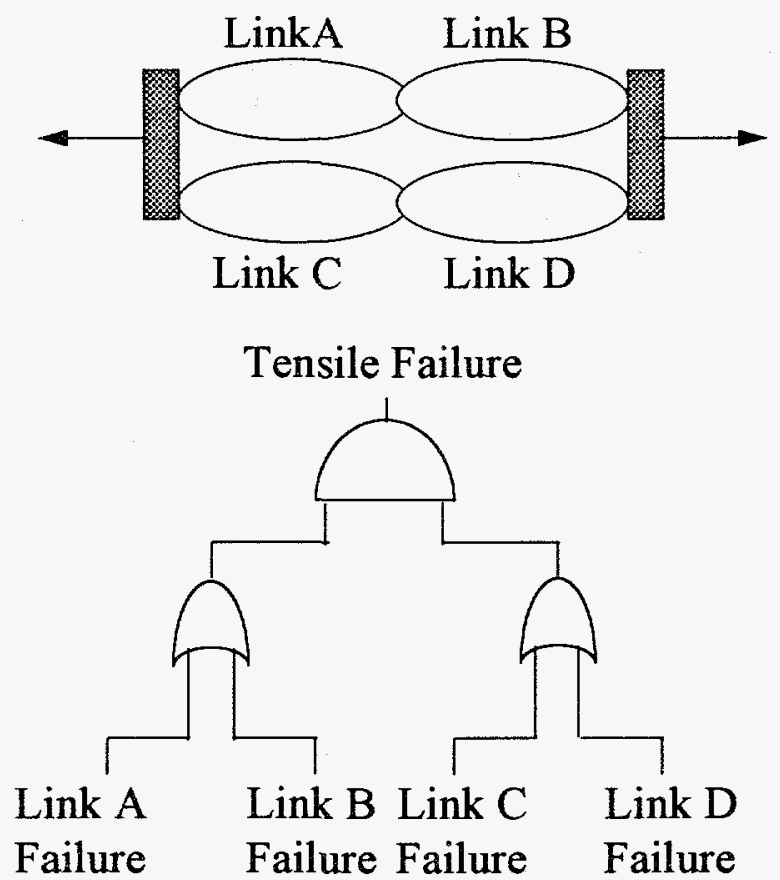

Figure 1. A Solution for Tensile Failure of Series-Parallel Chain Links

Below are shown example fuzzy function inputs for the failure characteristics of each link and the fuzzy logic combination to obtain the fuzzy output failure characteristic. Fuzzy 
"or" uses the maximum of the fuzzy operands; fuzzy "and" uses the minimum of the fuzzy operands. Mathematically, these operations are expressed as:

$$
\begin{aligned}
& \mu_{A \cup B}(x)=\mu_{A}(x) \vee \mu_{B}(x) \\
& \mu_{A \cap B}(x)=\mu_{A}(x) \wedge \mu_{B}(x)
\end{aligned}
$$

where $\mathrm{x}$ is the abscissa, the $\vee$ and $\wedge$ operators signify maximum ordinate value and minimum ordinate value, respectively, at each abscissa value, and:

$$
\mu_{A}(x) \in\{0,1\} \text { (membership value in the set } A \text { ). }
$$
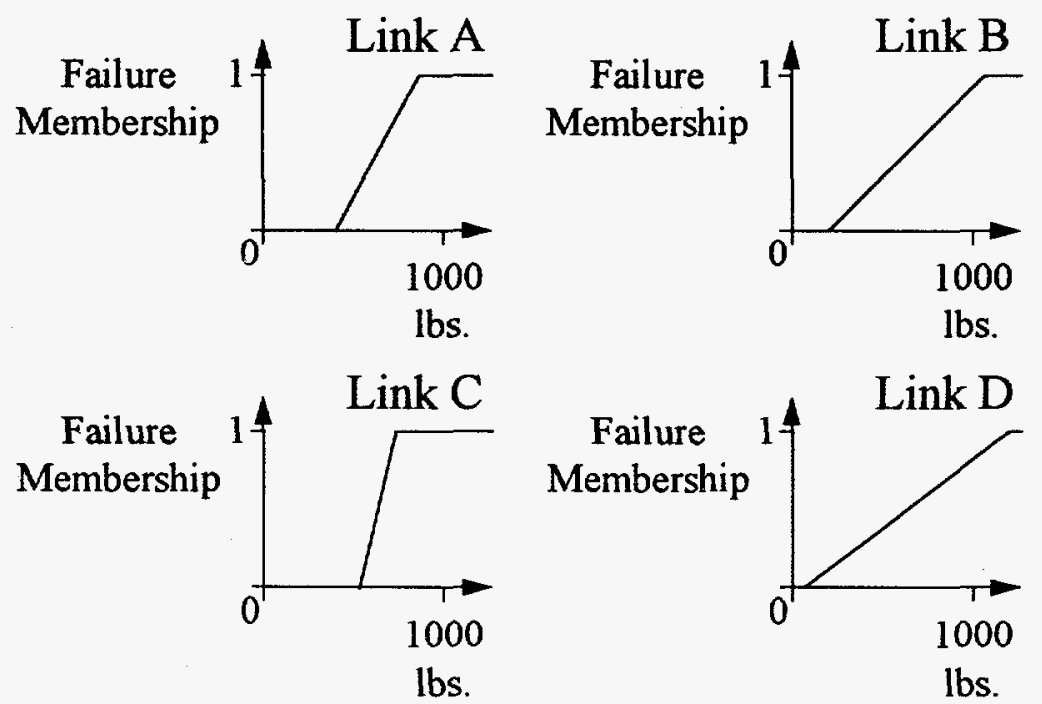

Figure 2. Subjective Failure Characteristics of Individual Links

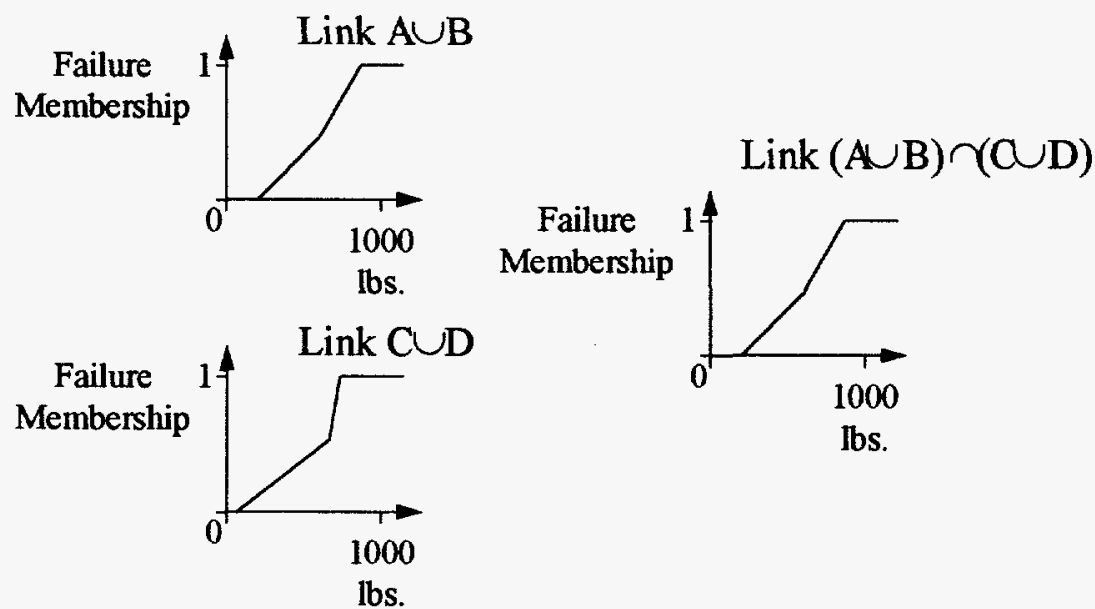

Figure 3. Fuzzy Logic Computation of Results 
Although this is a highly idealized problem, it is sufficient to illustrate the use of fuzzy logic.

We continue to investigate the potential applicability of fuzzy logic for PSA. However, the current version of PHASER does not utilize fuzzy logic. It incorporates fuzzy mathematics applied to "crisp" (conventional Boolean) logic constructs.

\section{Fuzzy Mathematics}

In contrast to fuzzy logic, fuzzy mathematics treats operands as fuzzy (subjectively known) numbers with uncertainty along the abscissa, and computes in terms of abscissa values rather than ordinate values. The general approach can be best illustrated through examples. The mathematical basis for combining fuzzy numbers is based on Zadeh's Extension Principle [Ref. 10]. For addition and multiplication (basic to fault tree and event tree computations), this produces:

$$
\begin{aligned}
& \mu_{A+B}(z)=\underset{z=x+y}{\vee}\left(\mu_{A}(x) \wedge \mu_{B}(y)\right) \\
& \mu_{A \times B}(z)=\underset{z=x \times y}{\bigvee}\left(\mu_{A}(x) \wedge \mu_{B}(y)\right)
\end{aligned}
$$

These are convolutions basically constructed like those used in probabilistic calculus. For logical consistency (and for mathematical operability), fuzzy numbers must be normal (having a maximum membership value of one) and convex (monotonically increasing with increases in abscissa values from zero to one and then monotonically decreasing from one to zero).

Although relations like those in Eqns. 4 and 5 are meaningful mathematically, there are more efficient ways to implement the operations in software [Ref. 11]. Under the conditions of greatest applicability to PSA, Eqns. 6 and 7 are mathematically equivalent to Eqns. 4 and $5^{2}$.

$$
\begin{aligned}
& A_{\mu}+B_{\mu}=\left[a_{1}(\mu)+b_{1}(\mu), a_{2}(\mu)+b_{2}(\mu)\right] \\
& A_{\mu} \times B_{\mu}=\left[a_{1}(\mu) \times b_{1}(\mu), a_{2}(\mu) \times b_{2}(\mu)\right]
\end{aligned}
$$

where the subscript 1 indicates the smallest abscissa value for a particular $\mu$, the subscript 2 indicates the largest value for a particular $\mu$, and the computation is done at both extremes for each $\mu$. The calculations in PHASER are based on Eqns. 6 and 7 (and similar equations for other necessary operations).

\footnotetext{
2 The mathematical equivalence of Eqns. 5 and 7 depends on restricting Eqn. 7 to nonnegative numbers. For probability numbers, this condition is met; for the more general situation, a more complex form of Eqn. 7 is required (described subsequently).
} 


\section{Interval Analysis}

For more comprehensive understanding of the mathematics implemented in PHASER, it is useful to consider the relation between interval analysis and fuzzy mathematics.

Interval processing is used when there is subjective or objective reason to specify only a lower bound and an upper bound over a continuous interval. Numbers are expressed as:

$$
X=\left[x_{1}, x_{2}\right] .
$$

Addition of two interval numbers is:

$$
A+B=\left[a_{1}, a_{2}\right]+\left[b_{1}, b_{2}\right]=\left[a_{1}+b_{1}, a_{2}+b_{2}\right] .
$$

In subtraction, the order of the pairs in the subtrahend must be inverted:

$$
A-B=\left[a_{1}-b_{2}, a_{2}-b_{1}\right] .
$$

Multiplication of nonnegative real numbers is specified by:

$$
A \times B=\left[a_{1} \times b_{1}, a_{2} \times b_{2}\right] .
$$

Multiplication of general real numbers is:

$$
A \times B=\left[\min \left(a_{1} b_{1}, a_{1} b_{2}, a_{2} b_{1}, a_{2} b_{2}\right), \max \left(a_{1} b_{1}, a_{1} b_{2}, a_{2} b_{1}, a_{2} b_{2}\right)\right] .
$$

Equation 12 is a solution for the general case mentioned earlier, a capability that is unnecessary when the numbers involved are probabilities. Since PHASER inputs and outputs are probabilities, Eqn. 11 is implemented in PHASER for multiplication.

The solution to Eqns. 6 and 7 for fuzzy operands is expressed in terms of interval operations at each $\mu$. PHASER incorporates up to $21 \mu$ "cuts" for each fuzzy number, so Eqns. 6 and 7 are solved by solving Eqns. 9 and 11 at each cut.

\section{Analysis of Extremes}

Lack of certainty about data affects the accuracy of variability determinations, especially at the extremes of the distribution of values. The main considerations at the extremes are accuracy and emphasis. Accuracy becomes a greater issue as data become more subjective; emphasis is a safety perspective. 


\section{Conventional Analysis of Extremes}

The conventional techniques for analysis of extremes [Ref. 12] include PMRM (Partitioned Multiobjective Risk Method) and Statistics of Extremes. The PMRM partitions data variability descriptions by a combination of probability of the occurrence and consequence. Greatest emphasis can be placed on low probability high consequence occurrences. The Statistics of Extremes takes advantage of theoretical work showing that one of a small family of asymptotic forms are likely to describe tail characteristics for a wide variety of situations. Both of these techniques give significant emphasis to the possibility of extreme values; neither gives as much emphasis as does fuzzy mathematics.

\section{Fuzzy Mathematics Analysis of Extremes}

Fuzzy mathematics gives a different perspective on extremes than conventional probabilistic analysis, because it incorporates subjectivity differently and because the mathematical operations involving each abscissa value are not constrained probabilistically by the corresponding ordinate values. For subjective inputs, there is no evidence to favor one approach over the other. However, for safety analyses, it is important to know that there are at least these two different perspectives about extremes.

Consider a "die-like" sextahedron (with six plane sides) having no other restriction on the possible shape. One form such an object might have is shown in Figure 4.

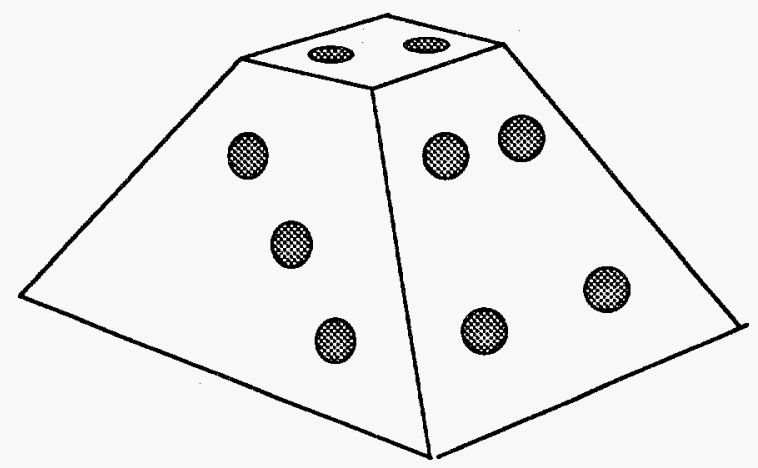

Figure 4. An Object with Six Plane Sides

The probability of throwing a particular number, say "one," using an unrestricted sextahedron is not knowable, but subjectively should be between zero and one-half. Suppose a solution for the probability of throwing four ones with four different such objects is desired. Using probabilistic calculus and a uniform distribution of values between zero and one-half, the solution for four ones is shown in Figure 5. 
The suppression of any indication that four ones might occur one sixteenth of the time is because a very low likelihood occurrence (probability $=1 / 2$ ) is modeled as extremely unlikely to occur four times. While this could be the case, it is not part of the given information; it is assumed to be the case. The dice could all be affected by the same flattening process, making the "one" outcome more likely, for example. The fuzzy mathematics approach is safer, in that it assumes less. A fuzzy ordinate for the probability of four ones is constant at one between zero and one sixteenth, because the fuzzy ordinate for any die selected is constant at one between zero and one half. The result is shown in

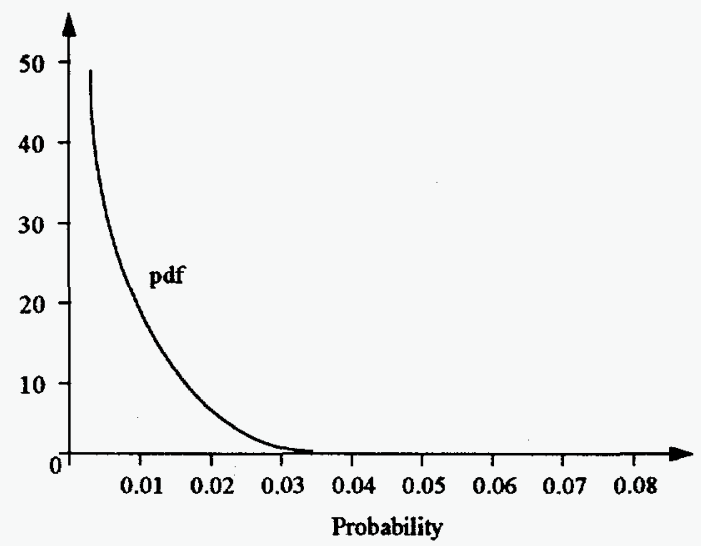

Figure 5. PDF for Probability of Throwing Four Ones

Figure 6. Note that more complex functional shapes could have been utilized for both the pdfs and the fuzzy estimates, but the tail suppression effect remains qualitatively the same.

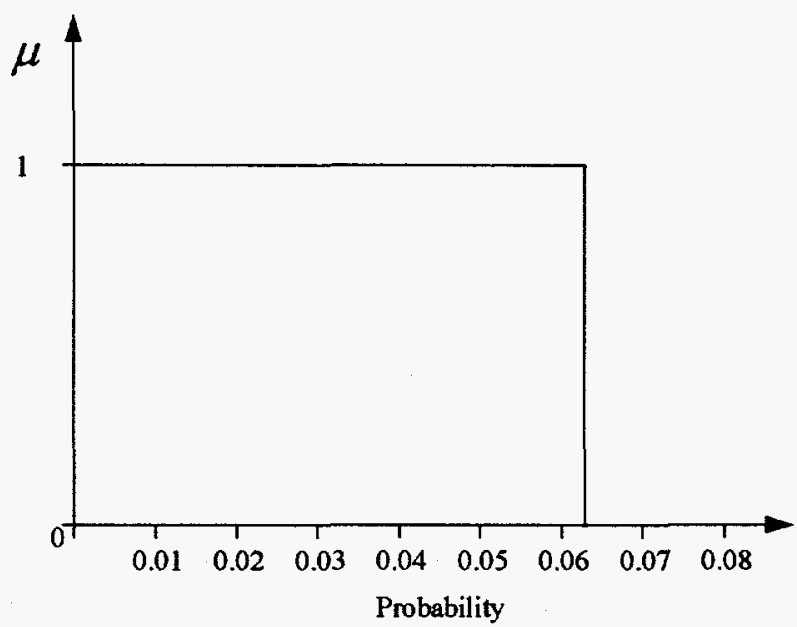

Figure 6. Subjective Estimate of Probability of Throwing Four Ones

PHASER currently displays extremes as part of the entire spectrum of uncertainty. Potential methods for emphasizing the regime of interest are being explored. 


\section{Hybrid Mathematics for Variability and Uncertainty}

Several years ago, Kaufmann and Gupta [Ref. 11] suggested that stochastic and subjective uncertainty could be considered together but separately in a pair ( $f, p)$, where $f$ is a fuzzy number and $\mathrm{p}$ is a pdf. These pairs, which they termed "hybrid numbers," can be added together by convolving the respective elements according to normal rules for fuzzy arithmetic and probability theory. The Kaufmann/Gupta formulation of hybrid numbers allows addition and subtraction. Addition is defined as:

$$
\left(f_{1}, p_{1}\right)+\left(f_{2}, p_{2}\right)=\left(f_{1}+f_{2}, p_{1}+p_{2}\right)
$$

where the plus signs on the right side of the equation represent fuzzy max-min convolution and ordinary probabilistic sum-product convolution, respectively. This formulation of hybrid numbers does not directly allow multiplication or a full hybrid arithmetic (e.g., the product of a completely fuzzy number and a completely probabilistic number is undefined). Nevertheless, the construct is useful, when modified slightly (explanation to follow).

\section{Scale Factors}

When the values of input variables are not well known, risk analysts may expect to improve their analyses by incorporating new information that is learned through additional tests, accident assessments, etc. In a Bayesian sense, stochastic information can be improved. Since new input data may only slightly improve the stochastic knowledge about ill-defined situations such as abnormal environment responses, a non-Bayesian hybrid analysis has a useful role. A reasonable approach to this problem that does not assume unavailable stochastic information is to provide for smooth transitioning from subjective (fuzzy) characterization to stochastic characterization as information about inputs is obtained.

1) Uniform Scale Factors. First, consider the case where the extent of knowledge about a problem is fractionally partitioned between stochastic and subjective portions. An input variable to an analysis whose variation characteristics are known partly stochastically and partly subjectively can be represented by a hybrid number with the relative stochastic/subjective information apportioned according to a scaling fraction:

$$
h(x)=a \times p(x)+(1-a) \times f(x)
$$

where $a$ is an estimated scale factor representing the fractional stochasticity of the overall knowledge $(0 \leq a \leq 1)$, and where $x$ and + are operators on $x$ values.

The scale factor is a scalar, which the form of Eqn. 14 suggests can be used to fractionally compress the abscissa (numeric) representation of the probabilistic constituent of

variability by $a$, along with compression of the fuzzy constituent of uncertainty by (1-a). 
The total variation is then additive along the abscissa, i.e., a scaled sum of the two constituents.

A visual description of the uncertainty represented by a scaled hybrid number is shown in Fig. 7, for an example scale factor of $1 / 2$. The axes represent the numeric variability due to the constituents of stochastic knowledge and subjective knowledge. The dashed indication of a fuzzy function has been scaled down by a factor of two along the $\mathrm{x}$ axis from the subjective estimate. The dot-dashed indication of a probability function has also been scaled down by a factor of two from the stochastic estimate. The $\mathrm{x}$-axis sum of the scaled variabilities is shown plotted as a three-dimensional hybrid number (solid lines).

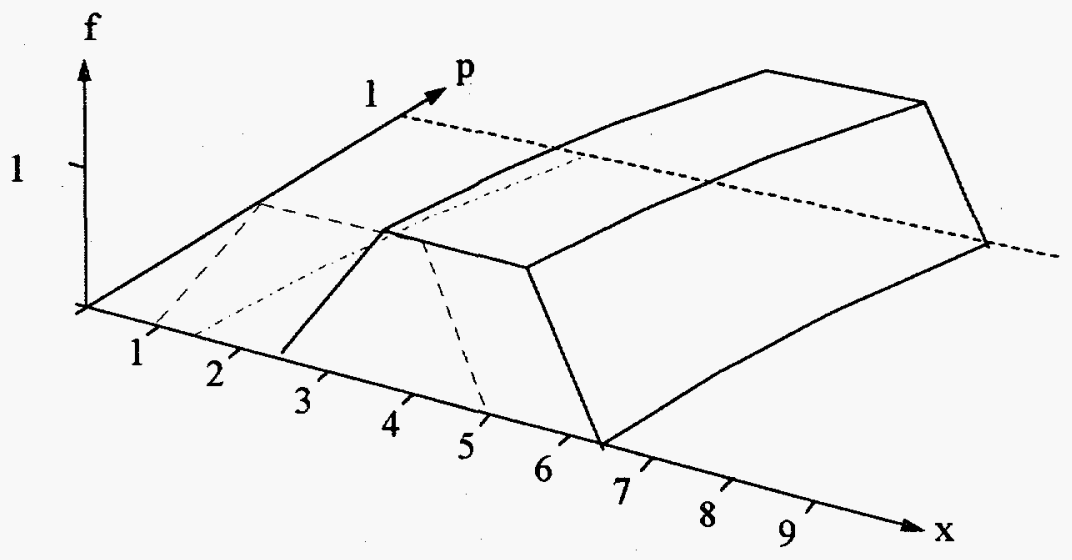

Figure 7. A Visual Depiction of a Scaled Hybrid Number

This formulation is understood most clearly if the spread and shape of the fuzzy function and the probability function do not interact with each other, and if separate stochastic and fuzzy mathematics are used. This is the approach implemented in PHASER.

In the limited case for which the scale factor applies uniformly to all input variables in a mathematical analysis (and therefore also to the output), the conventional mathematical properties (identities, commutative property, and associative property for addition and multiplication, and multiplication distributive over addition and subtraction) hold for scalefactor arithmetic with no further requirements ${ }^{3}$.

2) Individual Scale Factors. The more general case (allowing the scale factor to be individually chosen for each input operand), is of more practical interest. For this, a subscripted scale factor, $\mathrm{a}_{\mathrm{i}}$, will be introduced.

$$
h_{i}(x)=a_{i} \times p_{i}(x)+\left(1-a_{i}\right) \times f_{i}(x)
$$

\footnotetext{
${ }^{3}$ An important point is that variability for an operand must be entered only one time in equations in which there are multiple occurrences. For example, $A-A \equiv 0$. (We may be uncertain about the value of $A$, but not about the result of subtracting any value of $A$ from itself).
} 
As one way to maintain the desired mathematical properties and to also assure that results meet physical expectations, we choose to average operand scale factors to obtain a resultant scale factor for multiplication and to use an average weighted by the relative contribution of the operands for addition. The following binary operations illustrate the concept:

$$
\begin{gathered}
h_{1}+h_{2}=\frac{a_{1} \hat{p}_{1}+a_{2} \hat{p}_{2}}{\hat{p}_{1}+\hat{p}_{2}}\left(p_{1}(x)+p_{2}(x)\right)+\frac{\left(1-a_{1}\right) \hat{f}_{1}+\left(1-a_{2}\right) \hat{f}_{2}}{\hat{f}_{1}+\hat{f}_{2}}\left(f_{1}(x)+f_{2}(x)\right) \\
h_{1} \times h_{2}=\frac{a_{1}+a_{2}}{2}\left(p_{1}(x) \times p_{2}(x)\right)+\frac{\left(1-a_{1}+1-a_{2}\right)}{2}\left(f_{1}(x) \times f_{2}(x)\right)
\end{gathered}
$$

where $\hat{p}_{i}$ and $\hat{f}_{i}$ represent either point estimates of $p_{i}(x)$ and $f_{i}(x)$, respectively, or functions (probabilistic and fuzzy, respectively); the operations on the $p_{i}(x)$ are ordinary sum-product convolution; and the operations on the $\mathrm{f}_{\mathrm{i}}(\mathrm{x})$ are fuzzy max-min convolution. The use of the logic represented by Eqns. 16 and 17 assures that the desired mathematical properties (e.g., associativity, commutativity, distributivity) will be maintained.

Eqns. 16 and 17 can be generalized to $n$ operands ( $n>2$ ) by extending the number of subscripts in the equations from two to $n$. Alternatively, $n$ operands can be combined in successive binary operations by retaining information about prior operations. This is analogous to computing the average of the numbers $\mathrm{x}, \mathrm{y}$, and $\mathrm{z}$ by averaging $\mathrm{x}$ and $\mathrm{y}$, multiplying the result by 2 (number of previous operands), adding $z$ and dividing by 3 (total number of operands).

\section{Confidence Factors}

Note that if the additive weighting ratios are consistent (e.g., $\frac{\hat{p}_{1}}{\hat{p}_{1}+\hat{p}_{2}}=\frac{\hat{f}_{1}}{\hat{f}_{1}+\hat{f}_{2}}$ ), the scale factors resulting from mathematical operations sum to one. This is an interesting but unnecessary property, e.g., a hybrid number could have a well known fuzzy constituent and a well known stochastic constituent. The unrestricted equations are implemented in PHASER, and so the resultant scale factors are termed "confidence factors."

The attribute of confidence factors is that they provide a metric for the amount of relative knowledge about stochastic information and subjective information, supplementing the indications about the total amount of stochastic variability and subjective uncertainty shown qualitatively. 


\section{Disjoint Subsets}

The "top event" of a fault tree or fault tree/event tree combination, T, is typically expressed as the Boolean sum of "cutsets," $\mathrm{C}_{\mathfrak{j}}$, where each cutset is an intersection (Boolean "and") of basic events, $e_{\mathrm{i}}$. For example,

$$
T=C_{1} U C_{2} U \ldots U C_{n},
$$

where $C_{1}=e_{11} e_{12} \ldots e_{1 m_{1}}, C_{2}=e_{21} e_{22} \ldots e_{2 m_{2}}$, etc.

It is often necessary to solve for the probability of the top event, based on knowledge of the probabilities of the basic events. The cutset probabilities can be obtained through algebraic multiplication of the basic event probabilities if the basic events are independent, and seeking independent basic events is central to the logical approach. The top event probability can be computed as the algebraic sum of the cutsets only if the cutsets are disjoint, but in practical problems cutsets are almost never disjoint.

This problem is frequently handled by a "rare-event approximation," meaning that the cutset probabilities are often small enough that the error resulting from the lack of disjointness is small. However, for many practical problems, this assumption is not appropriate, and in fact for these problems, the error in the top event probability can be significant, the computed value even exceeding one (impossible for true probability numbers).

Another technique that partially circumvents the above problem is to compute a "corrected" sum for the most significant terms by using the relation:

$$
P(A U B)=P(A)+P(B)-P(A) P(B)
$$

The disadvantages of this approach are that the above result is only accurate if the involved cutsets have no common basic events, and whether or not this condition is met, the final result is still not correct, since all cutsets are not involved in the computation.

An approach based on the above equation can be used to compute an exact answer by carefully accounting for the effect of common basic events and applying the procedure to all cutsets, but this approach is excessively complex for most practical problems. In particular, it requires keeping track of nearly $2^{\mathrm{n}}$ entities for a problem having $\mathrm{n}$ cutsets. If $\mathrm{n}$ is as great as 100 (a common size), the memory requirements become so great that processing can be done only at the expense of reasonable computation time. Because of the memory and time required, exact computations for reasonably large problems are usually not attempted. 
The following procedure, implemented in PHASER, solves the above problems. It gives an exact answer, whether or not cutsets are disjoint and whether or not they contain common basic events. It takes advantage of a unique recursive mathematical approach to assure an efficient solution. The description of the procedure follows.

Consider $\mathrm{T}=\mathrm{C}_{1} \mathrm{U} \mathrm{C}_{2} \mathrm{U} \ldots \mathrm{U} \mathrm{C}_{\mathrm{n}}$, where $\mathrm{T}$ is the top event and the $\mathrm{C}_{\mathrm{i}}$ are the cutsets. The requirement is to use arithmetic operations to compute $\mathrm{T}$. Another constraint is to be efficient in computer time and memory.

The basis of the algorithm is to note that:

$$
T=C_{1}+C_{2} \bar{C}_{1}+C_{3} \bar{C}_{2} \bar{C}_{1}+\ldots+C_{n} \bar{C}_{n-1} \ldots \bar{C}_{1},
$$

where + is the appropriate operation, because the sets are disjoint. By "DeMorgan's theorem," $\bar{C}_{i}=\overline{\boldsymbol{e}}_{i 1} U \overline{\boldsymbol{e}}_{i 2} U \ldots U \overline{\boldsymbol{e}}_{i m_{4}}$. The result of the required Boolean processing is disjoint sets comprising basic events. The order in which the processing is done has no logical significance, but is extremely important to maximize efficiency.

Two operations dramatically improve efficiency for significant-sized problems. One of these is the first step in the procedure. The second is the complexity indicator, which is repeated as a basic part of the recursive procedure. The procedure steps are:

1. Any group of basic events that occur only together are given a substitute name for processing by the procedure. They are treated in the procedure as a single variable, although these substitute variables are subsequently replaced by the basic events for the final top event computation.

2. The order of the cutsets is determined by the complexity indicator, essentially indicating how many new terms will be generated by the procedure at each step. The computation for cutset " $\mathrm{i}$ " is the sum of the other cutsets in which variable " $\mathrm{j}$ " does not occur, summed over all variables in cutset $i$. The cutset with the minimum computed value is used as $C_{1}$. A tie is resolved in favor of the cutset with the fewest variables (minimizing the number of compares required).

3. $\mathrm{C}_{1}$ is selected as the first disjoint set. It becomes a "compare reference" for each of the remaining cutsets. The comparison is used to determine new "candidates" for the disjoint cutset collection. The candidates are modified forms of the original cutsets.

4. The comparison is made in order to incorporate the intersection of each cutset with $\bar{C}_{1}$ (see Eq. 20). Since $C_{1}=e_{11} e_{12} \ldots e_{1 m_{1}}, \bar{C}_{1}=\bar{e}_{1}+\bar{e}_{2}+\ldots+\bar{e}_{m_{1}}$, by DeMorgan's theorem. This means that the intersection with $\bar{C}_{1}$ can be performed by intersecting with the complements of each of the basic events in $C_{1}$. This must 
be done for each of the other cutsets to obtain candidates for the next disjoint set selection.

5. The new candidates are then subjected to a complexity indicator computation, exactly like the original cutsets were. The candidate with the lowest complexity computation becomes the next disjoint set member.

6. $\mathrm{C}_{2}$ from the new disjoint set member then takes on the role of $\mathrm{C}_{1}$ in repeating steps 3, 4, and 5, and the remaining candidates take on the role of the "remaining cutsets." This iteration is repeated until there are no remaining candidates.

An example illustrates how the procedure works. Boolean minimization is used throughout. The example top event equation is:

$$
\mathrm{T}=\mathrm{ABC} U \mathrm{ABD} U \mathrm{CDE} \mathrm{U} \text { ABFGH }
$$

Step 1: $A B=X ; F G H=Y$; so $T=X C$ U XD U CDE U XY

Step 2:

$\begin{array}{ccccc} & \mathrm{XC} & \mathrm{XD} & \mathrm{CDE} & \mathrm{XY} \\ \mathrm{X} & \mathrm{x} & \mathrm{x} & & \mathrm{x} \\ \mathrm{C} & \mathrm{x} & & \mathrm{x} & \\ \mathrm{D} & & \mathrm{x} & \mathrm{x} & \\ \mathrm{E} & & & \mathrm{x} & \\ \mathrm{Y} & & & & \mathrm{x}\end{array}$

The computations are (counting blanks): for $\mathrm{XC}, 3$; for $\mathrm{XD}, 3$; for $\mathrm{CDE}, 7$; for $\mathrm{XY}, 4$.

Step 3: XC becomes the first disjoint set.

Step 4: The intersection of $\overline{X C}$ with the other cutsets is used to determine the candidates: $X \bar{C} D, \bar{C} X Y$, and $C D E \bar{X}$.

Step 5:

$$
X \bar{C} D \bar{C} X Y \quad C D E \bar{X}
$$

$\begin{array}{llll}\mathrm{X} & \mathrm{x} & \mathrm{x} & \\ \bar{C} & \mathrm{x} & \mathrm{x} & \\ \mathrm{D} & \mathrm{x} & & \mathrm{x} \\ \mathrm{Y} & & \mathrm{x} & \\ \mathrm{C} & & & \mathrm{x} \\ \mathrm{E} & & & \mathrm{x} \\ \bar{X} & & & \mathrm{x}\end{array}$


The computations are: for $X \bar{C} D, 3$; for $\bar{C} X Y, 4$; for $C D E \bar{X}, 7$.

Step 3 (second pass): $X \bar{C} D$ becomes the second disjoint set.

Step 4 (second pass): New candidates are determined by intersecting $\overline{X D}$ with the other previous candidates to obtain: $\bar{C} \bar{D} X Y$ and $C D E \bar{X}$.

Step 5 (second pass):

$\begin{array}{ccc} & \bar{C} \bar{D} X Y & C D E \bar{X} \\ \bar{C} & \mathbf{x} & \\ \bar{D} & \mathbf{x} & \\ \mathrm{X} & \mathbf{x} & \\ \mathrm{Y} & \mathrm{x} & \\ \mathrm{C} & & \mathrm{x} \\ \mathrm{D} & & \mathrm{x} \\ \mathrm{E} & & \mathrm{x} \\ \bar{X} & & \mathrm{x}\end{array}$

The computations are: for $\bar{C} \bar{D} X Y, 4$; for $C D E \bar{X}$, 4. The first (two compare variables) is chosen.

Step 3 (third pass): $\bar{C} \bar{D} X Y$ becomes the third disjoint set.

Step 4 (third pass): $\overline{X Y}$ is intersected with the other previous candidate to obtain new candidate: $C D E \bar{X}$. This is the last disjoint set.

The result is:

$$
T=C X+\bar{C} D X+\bar{C} \bar{D} X Y+C D E \bar{X}
$$

Replacing the substitute variables, we obtain the final answer:

$$
T=A B C+A B \bar{C} D+A B \bar{C} \bar{D} F G H+\bar{A} C D E+A \bar{B} C D E \quad .
$$

\section{Constrained Mathematics}

The mathematics of uncertainty has some subtle requirements. As an example, consider the probabilistic "or" of fuzzy inputs. The computation of the probability as an "or" function of inputs $A$ and $B$, for which probabilistic estimates are available, is: 


$$
P(A \cup B)=P(A)+P(B)-P(A) P(B)
$$

The "or" is shown on the left side of the equation, the terms on the right are computed with ordinary addition, subtraction, and multiplication, and the final subtraction is necessary because it is included in each of the previous two terms, and therefore is counted once too much. All of the operations on the right side can be done with fuzzy arithmetic, but extreme care is necessary in order to do the problem correctly. The subtlety is that as each individual operation is performed (at a particular level of presumption and direction), the above equation must be used as if the variables were crisp (ordinary) at that level of presumption. This cannot be accomplished by fuzzy addition, fuzzy multiplication, and fuzzy subtraction done separately, because the above equation is a crisp equation. Therefore, the fuzzy "or" computation cannot be segmented. This is a "constrained" fuzzy operation (there are many other examples of such constrained operations). Without the constraint, it is possible to get "probabilities" less than zero or greater than one. As an example, consider the problem below, where we are to compute the probability of A "or" B by adding the probabilities and subtracting the "and." Suppose we solved Eqn. 21 in what might appear to be the "obvious" way--by adding the fuzzy numbers $P(A)$ and $P(B)$ and subtracting the fuzzy number $P(A \times B)$. The result is an "unconstrained" solution, for which three of the solution points are indicated in Figure 8 by Xs. Clearly, there is a problem with this solution, because it gives "probabilities" less than zero and greater than one. The problem lies in the lack of constraint for a problem which is constrained by definition: The dashed line corresponds to the correct constrained operation. If more than two variables are "ored," the above procedure can be applied iteratively.

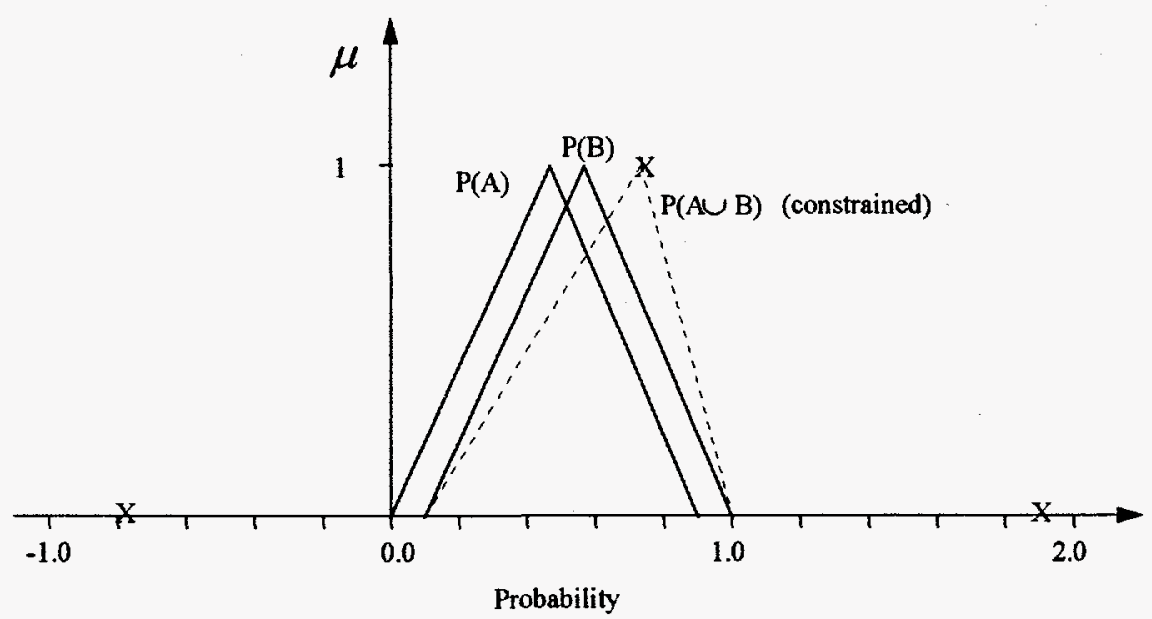

Figure 8. Example of Constrained Mathematics Result for Probability of Union of Two Events

Constrained uncertainty mathematics are implemented in PHASER. The union of cutsets would be sufficient to require constrained mathematics, as outlined above. In addition, the 
disjoint set solution requires constrained mathematics, because of the presence of negated events. The inverse of a probabilistic event input is one minus the probability of the event. The negation would ordinarily require inverting the interval bounds, as illustrated in Equation 10. Since all negated events are accompanied in the top event equation by unnegated events, the constrained operation requires only that the interval limits not be inverted. Since it is expected that PHASER inputs will not include negated events without corresponding unnegated events, this relatively straightforward solution to the constrained mathematics requirement is sufficient.

\section{Dependence Among Inputs}

The common assumption of independent inputs is very difficult to achieve in weapon safety problems. As a partial response to this problem, there are capabilities built into some codes, such as LHS, that can accommodate measures of correlation. Since correlation is only a subset of dependence, it is helpful in weapon safety analysis to pursue other more comprehensive approaches. One such approach was found applicable to the types of problems for which PHASER is intended. This is called "Dependency Bounds Analysis," or DBA [Ref 13].

The basis for DBA is recognition that dependence of inputs for any form of analysis gives results that are bounded by the completely independent case on one extreme, and the value representing the most severe deviation from independence on the other extreme.

Mathematical operations following this concept are based on the classical Frechet bounds. Although PHASER Version 1.00 has no dependence capabilities built in, subsequent versions will allow an optional user input to reflect subjective judgment of the position in the range of dependence values specified by the Frechet bounds. These can be entered for basic events or for the top event. In each case, the Frechet bounds will be displayed as well as any user-selected dependence between bounds.

\section{Displaying Results}

\section{Segmented Plots}

PHASER output is in the form of segmented (separate stochastic and subjective) uncertainty plots. The user is given side-by-side displays of stochastic variability and subjective uncertainty, along with the corresponding confidence factors. No interpretation is made; the deduction of meaning is a user prerogative. In future versions of PHASER, different forms of output, such as three-dimensional combinations of stochastic and subjective information may be considered. 


\section{Three-Dimensional Plots}

A hybrid number can be formulated as a cumulative probability distribution function (cdf) that is only fuzzily known. A formal definition depends on monotonicity of the cdfs and nestedness of the fuzzy functions. Let $\mathbf{P}$ denote the set of cdfs defined on the real numbers $\mathbf{R}$, so each element $p \in \mathbf{P}$ is a surjective function $p: R \rightarrow[0,1]$ such that $p\left(x_{1}\right) \leq p\left(x_{2}\right)$ whenever $x_{1}<x_{2}$. A hybrid number can be defined as a set of closed intervals, each characterized by a pair of functions from $\mathbf{P}$ :

$$
\mathbf{H}:[0,1] \rightarrow \mathbf{P} \times \mathbf{P}: \alpha \mapsto\left(\mathrm{p}_{\alpha}, \mathrm{q}_{\alpha}\right)
$$

such that for $\alpha, \beta \in[0,1], p_{\alpha}(x) \geq p_{\beta}(x) \geq q_{\beta}(x) \geq q_{\alpha}(x)$ whenever $\alpha<\beta$. Here, $\alpha$ and $\beta$ represent fuzzy membership values $(\alpha, \beta$ cuts) for values of $x$. The collection of cuts

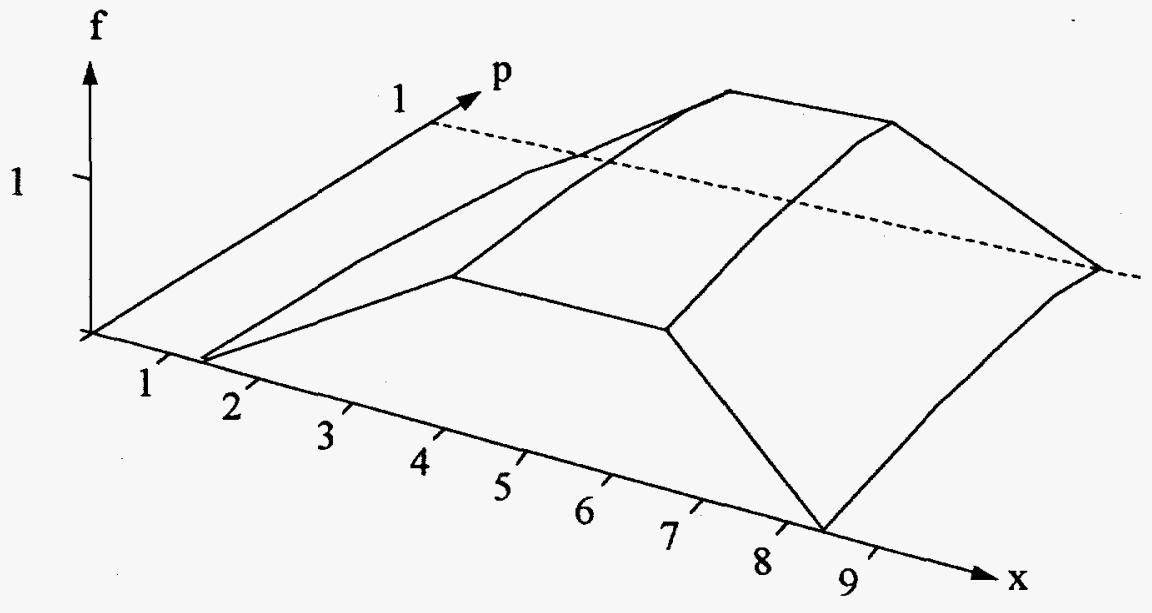

Figure 9. Example Three-Dimensional Image of a Hybrid Number

across $p(x)$ is from the set $\mathbf{F}$ of fuzzy numbers. In Figure 9, cdfs $p(x)$ and fuzzy uncertainty functions $\mathrm{f}(\mathrm{x})$ fall naturally into a three-dimensional form. When fuzzy functions can be represented by a few key vertices (e.g., three for triangular fuzzy numbers, four for trapezoidal fuzzy numbers), the two-dimensional view of the threedimensional form of Figure 9 taken along the f axis represents sufficient information without using the third dimension. This two-dimensional view is useful for computer routine output displays. The concept is illustrated in Fig. 10, where the four lines shown are loci for the four vertices of the trapezoidal fuzzy numbers in Figure 9. 


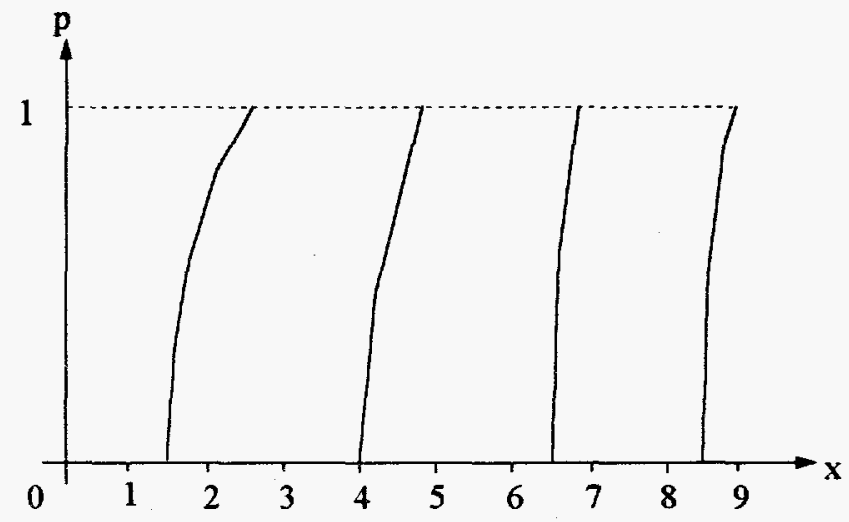

Figure 10. Two-Dimensional Representation of Fig. 9

Version 1.00 of PHASER does not use either of these output display strategies. They are presented here because they aid in understanding the hybrid processing used in PHASER and because subsequent versions of PHASER may use different forms of output. The formulation described in this section is mathematically equivalent to one based on fuzzy numbers and pdfs. However, due to lack of monotonicity of pdfs, the graphical images for hybrid numbers lack the appealing simplicity inherent in the cdfs. 


\section{References}

1. Ernest J. Henley and Hiromitsu Kumamoto, Probabilistic Risk Assessment, IEEE Press, 1992.

2. Max Henrion and B. Fischhoff, "Assessing Uncertainty in Physical Constants," American Journal of Physics, 54, No. 9 (September: pp. 791-798.

3. M. Granger Morgan and Max Henrion, Uncertainty, pp. 57-59, Cambridge University Press, 1990.

4. Alexander Shlyakhter, "An Improved Framework for Uncertainty Analysis: Accounting for Unsuspected Errors," Risk Analysis, Vol. 14, No. 4, 1994.

5. Dale Hattis and David Burmaster, "Assessment of Variability and Uncertainty Distributions for Practical Risk Analyses," Risk Analysis, Vol. 14, No. 5, 1994.

6. R. E. Follensbee, "The Fail Safe Concept," Seattle Aircraft Certification Office Systems Designated Engineering Representative Workshop, Sept. 14, 1993.

7. Roger Breeding, J. C. Helton, and E. D. Gorham, "Summary Description of the Methods used in the Probabilistic Risk Assessments for NUREG-1 150," Nuclear Engineering and Design 135: pp.1-27.

8. A. A. Sveshnikov, Problems in Probability Theory, Mathematical Statistics, and Theory of Random Functions, Dover Publications Inc., 1968.

9. Thomas L. Saaty and Luis G. Varga, The Logic of Priorities, RWS Publications, 1991.

10. Timothy J. Ross, Fuzzy Logic with Engineering Applications, McGraw-Hill, Inc., 1995.

11. Arnold Kaufmann and Madan Gupta, Introduction to Fuzzy Arithmetic, Van Nostrand Reinhold, 1991.

12. A. H. S. Ang and W. H. Tang, Probability Concepts in Engineering Planning and Design, Vol. II, John Wiley and Sons, 1984.

13. S. Ferson, L. R. Ginzburg, and H. R. Akcakaya, "Whereof One Cannot Speak: When Input Distributions are Unknown," Risk Analysis [in press]. 
Distribution:

W. R. Reynolds, 2103 MS 0427

P. N. Demmie, 2415 MS 0303

C. F. Briner, 2674 MS 0328

M. J. DeSpain, 2671 MS 0311

D. M. Kunsman, 5417 MS 0423

A. C. Payne, Jr., 5415 MS 0425

W. R. Burcham, 2123 MS 0485

K. Ortiz, 2102 MS 0435

C. B. Richardson, 5417 MS 0423

M. S. Elpel, 2254 MS 9036

L. R. Gilliom, 5133, MS 0458

P. E. Rexroth, 5209 MS 1393

R. T. Heaphy, 5521 MS 0763

A. L. Camp, 6412 MS 0747

S. L. Daniel, 6412 MS 0747

G. D. Wyss, 6412 MS 0747

M. J. Martinez, 6613 MS 0746

S. Neuhauser, 6641 MS 0718

R. H. Yoshimura, 6641 MS 0718

Technical Library, 4414 MS0899 (5)

Print Media, 12615, MS0619

G. Pirtle, 7316 MS 1036

Document Processing for DOE/OSTI, 7613-2 MS 0100 (2)

J. T. Ringland, 8112 MS 9201

T. E. DeLano, 8114 MS 9201

R. Zurn, 8114 MS 9201

C. W. Pretzel, 8414 MS 9408

Central Technical Files, 8523-2 MS 9018

Judy Lim, 8910 MS 9011

K. M. Simonson, 2533 MS 0844

W. C. Fan, 9352 MS 1166

L. F. Restrepo, 9364 MS 1146

S. K. Fletcher, 9411 MS 0777

M. Murphy, 9411 MS 0777

R. M. Jansma, 9415 MS 0777

W. C. Nickell, 12300 MS 0631

T. S. Edrington, 12301 MS 0632

R. G. Easterling, 12302 MS 0829

R. E. Smith, 12302 MS 0491

V. J. Johson, 12304 MS 0621

K. V. Diegert, 12323 MS 0829

P. E. D'Antonio, 12324 MS 0491

J. P. Berry, 12324 MS 0491 
M. Caldwell, 12324 MS 0491

J. M. Covan, 12324 MS 0491

L. W. Dahlke, 12324 MS 0491

M. E. Ekman, 12324 MS 0491

D. Isbell, 12324 MS 0491

J. Leyland, 12324 MS 0491

R. D. Pedersen, 12324 MS 0491

L. Vaughn, 12324 MS 0491

P. W. Werner, 12324 MS 0491

K. S. Ricker, 12326, MS 0638

S. D. Spray, 12331 MS 0490

R. R. Bennett, 12331 MS 0490

J. A. Cooper, 12331 MS 0490 (20)

J. P. Hoffman, Jr. , 12331 MS 0490

W. E. Mauldin, 12331 MS 0490

C. A. Trauth, Jr. , 12331 MS 0490

G. A. Sanders, 12332 MS 0492

J. P. Cates, 12332 MS 0492

P. J. Konnick, 12332 MS 0492

D. R. Lewis, 12332 MS 0492

D. H. Loescher, 12332 MS 0492

D. R. Olson, 12332 MS 0492

C. G. Shirley, 12332 MS 0492

D. A. Summers, 12332 MS 0492

J. F. Wolcott, 12332 MS 0492

D. D. Carlson, 12333 MS 0405

D. E. Bennett, 12333 MS 0405

R. J. Breeding, 12333 MS 0405

M. A. Dvorack, 12333 MS 0405

M. K. Fuentes, 12333 MS 0405

N. R. Grandjean, 12333 MS 0405

J. V. Hancock, 12333 MS 0405

T. R. Jones, 12333 MS 0405

S. A. Kalemba, 12333 MS 0405

Y. T. Lin, 12333 MS 0405

W. McCulloch, 12333 MS 0405

K. J. Maloney, 12333 MS 0405

R. J. Roginski, 12333 MS 0405

K. B. Sobolik, 12333 MS 0405

J. L. Tenney, 12333 MS 0491

R. O. Winchester, 12333 MS 0405

G. C. Novotny, 12334 MS 0627

S. Humbert, 12334 MS 0627

E. L. Fronczak, 12334 MS 0627

J. E. Stayton, 12334 MS 0627 
F. G. Trussell, 12334 MS 0627

J. M. Sjulin, 12335 MS 0830

D. S. Hill, 12367 MS 0636

P. D. Sands, 4621 MS 0811

Lt. Col. Dave Grenda

Field Command, Defense Nuclear Agency

FCFA

Kirtland AFB NM 87115-5000

Lt. Col. John Waskiewicz

HQ AFSA?SEWA

Kirtland AFB NM 87117-5000

Col. John R. Curry

SA-ALC/NWI

1651 First St. SE

Kirtland AFB NM 87117-5617

Harvey Dayhoff

SA-ALC/NWIS

1651 First St. SE

Kirtland AFB NM 87117-5617

Al Matteucci

SA-ALC/NWIE

1651 First St. SE

Kirtland AFB NM 87117-5617

Prof. Nancy Leveson

Computer Science Engineering Department

University of Washington

Seattle WA 98195

Dr. Scott Ferson

Applied Biomathematics

100 North Country Road

Setauket, NY 11733

Prof. Lev Ginzburg

Ecology and Evolution

State University of New York

Stony Brook, NY 11794 